\title{
Cementáa
}

\section{Adesão da elite brasileira de pesquisadores aos periódicos de acesso aberto: a relação com gênero, região geográfica e grande área do conhecimento}

\author{
Elaine Hipólito dos Santos Costa \\ Doutora; Universidade Federal de São Paulo, São Paulo, SP, Brasil; \\ elaine.hipolito@unifesp.br \\ Simone da Rocha Weitzel \\ Doutora; Universidade Federal do Estado do Rio de Janeiro, Rio de Janeiro, RJ, Brasil; \\ sweitzel@unirio.br \\ Jacqueline Leta \\ Doutora; Universidade Federal do Rio de Janeiro, Rio de Janeiro, RJ, Brasil; \\ jleta@bioqmed.ufrj.br
}

\begin{abstract}
Resumo: O presente estudo tem por objetivo avaliar o nível de adesão da elite brasileira de pesquisadores aos periódicos de acesso aberto como estratégia para publicar suas pesquisas. Neste estudo, a elite foi definida como o grupo de 1.205 pesquisadores que recebiam bolsa de produtividade PQ 1A do CNPq em 2016. Foram coletados, na Plataforma Lattes, dados pessoais e acadêmicos e dados sobre a produção científica desses pesquisadores referentes ao período de 2000 a 2015, em quadriênios. Definiu-se adesão como a fração de artigos em acesso aberto de cada um dos 1.205 pesquisadores PQ 1A em relação ao total de artigos que eles publicaram. Foram realizadas três análises, buscando identificar a relação da adesão aos periódicos de acesso aberto com: (1) o gênero dos pesquisadores, (2) a região geográfica da instituição de vínculo e (3) a grande área de pesquisa definida pelo CNPq. Verificou-se, ao longo dos quadriênios, uma redução na faixa de pesquisadores classificados como de muito baixa adesão ao acesso aberto, porém, somente $10 \%$ dos pesquisadores foram classificados como de alta ou muito alta adesão. Pesquisadoras mulheres, pesquisadores da região Nordeste e pesquisadores das ciências agrárias e das ciências sociais aplicadas mostraram maior adesão aos periódicos de acesso aberto. O conjunto de resultados indica que a adesão a periódicos de acesso aberto pela elite brasileira de pesquisadores, apesar de crescente, ainda é baixa, um cenário preocupante, dado que esse grupo é referência para a comunidade científica brasileira.
\end{abstract}

Palavras-chave: Acesso aberto. Periódicos científicos. Ciência brasileira. ScriptLattes. Produção Científica. 


\section{Introdução}

A proposta original do Acesso Aberto (AA), estabelecida na Declaração de Budapeste (Budapest Open Access Initiative) no início do século XXI, consistiu em disponibilizar a produção científica em texto completo proveniente de periódicos revisados por pares sem barreiras de acesso e de forma gratuita, o que seria alcançado por meio de duas estratégias: acesso aberto verde e acesso aberto dourado (OPEN SOCIETY INSTITUTE, c2001). A primeira está baseada no depósito de artigos publicados em periódicos de acesso restrito em repositórios institucionais ou temáticos para potencializar a visibilidade e a democratização do acesso ao conhecimento científico. Em alguns casos, devido às restrições dos editores comerciais, é possível depositar o artigo em repositórios institucionais ou temáticos em sua versão original, isto é, sem a revisão de pares. Esta é uma alternativa de acesso até que se possa fazer o depósito do texto em sua versão completa, após o período de embargo. Já a segunda estratégia, o acesso aberto dourado, está baseada na produção de uma nova geração de periódicos científicos, que não cobram dos autores para publicar artigos nem dos leitores para acessá-los, ao contrário dos periódicos de editoras comerciais.

Para os defensores do AA, uma vez consolidadas estas duas estratégias, os resultados das pesquisas, principalmente aquelas financiadas com recursos públicos, passariam a ter condições de ser disseminados amplamente, não apenas entre cientistas e pesquisadores, mas também para novos e diversos públicos. A ampla disseminação dos resultados das pesquisas poderia, assim, promover tanto o efetivo uso da literatura para buscar as respostas que poderão solucionar os problemas da humanidade como a prestação de contas à sociedade.

A despeito do benefício implícito do AA para a ciência global, muitos desafios ainda precisam ser superados para que o acesso aberto seja adotado de forma plena. Dentre os inúmeros fatores que estão em jogo, desde os econômicos e políticos até os institucionais, há também aqueles relativos à escolha dos periódicos para divulgação dos resultados das pesquisas pelos 
pesquisadores. Em revisão de literatura sobre o tema, Björk e Holmström (2006) propuseram um modelo com quatro eixos centrais que traduz as principais razões que determinam essa escolha: desempenho (incluindo taxa de rejeição, tempo de publicação, entre outros aspectos), prestígio (fator de impacto, qualificação do corpo editorial, entre outros aspectos), audiência (número de assinantes, número de downloads, entre outros aspectos) e infraestrutura (valores das taxas de assinatura e outras características técnicas, entre outros aspectos).

Estudos posteriores revelaram que a maior parte dos autores leva em consideração aspectos referentes a uma ou mais razões apresentadas no modelo, sendo o prestígio e a audiência aquelas de maior frequência, tal como observaram Bröchner e Björk (2008), Cheung (2008), Pepermans e Rousseau (2016), Nicholas e colaboradores (2017) e Boukacem-Zegmouri e outros (2018).

Esses estudos constataram que poucos pesquisadores ou nenhum considerou o acesso aberto como um critério para publicar. É possível que este resultado ainda seja um reflexo do desconhecimento das estratégias do AA (MOORE, 2011; SHEIKH, 2017) ou de uma percepção negativa associada à falta de prestígio ou de credibilidade de periódicos de AA (SCHROTER; TITE; SMITH, 2005; GUMPENBERGER; OVALLE-PERANDONES; GORRAIZ, 2013; RODRIGUEZ, 2014). Essa última explicação é, aparentemente, mais frequente entre os pesquisadores mais experientes (TENOPIR, 2017) e, de certa forma, contrasta com estudos recentes que identificam um número maior de citações para os pesquisadores que publicam em AA, garantindo, assim, maior visibilidade e impacto (LARIVIÈRE; HAUSTEIN; MONGEON, 2015; ARCHAMBAULT et al., 2016). Esse efeito positivo de aumentar a visibilidade do trabalho quando se publica em periódicos de acesso aberto é uma das vantagens que o movimento do acesso aberto vem defendendo e demonstrando desde o início da década de 2000.

Outro fator que dificulta a ampla adoção do AA é a ausência do tema nos programas de política científica dos países ou a demora com que ele é incluído nesses programas. Atento à urgência da matéria Sander Dekker, Secretário de Educação, Cultura e Ciência dos Países Baixos declarou 
Pesquisa e inovação geram crescimento econômico e emprego e também soluções para os desafios da sociedade. [...] a Europa precisa ser mais atrativa para os pesquisadores [...]. Isso exige que o conhecimento seja livremente compartilhado. O momento para falar em acesso aberto já passou. (KHOMAMI, 2016, doc. não paginado, tradução nossa).

No caso do Sul Global, países como México, Argentina e Peru, por exemplo, regulamentaram por meio de leis específicas o desenvolvimento de mecanismos nacionais para implementar o acesso aberto. Mesmo sem uma legislação dedicada ao acesso aberto para além das teses e dissertações (BRASIL, 2006), o Brasil foi pioneiro em várias ações que contaram com o apoio direto do Estado brasileiro. Modelos de boas práticas foram desenvolvidos como as plataformas Scientific Electronic Library Online (SciELO) e as estratégias do Instituto Brasileiro de Informação em Ciência e Tecnologia (IBICT) para ampliar a adoção do software Sistema Eletrônico de Editoração de Revistas (SEER) entre instituições de pesquisa e de ensino superior), assim como o fomento do acesso aberto dourado na América Latina têm garantido ao Brasil certa liderança em conteúdo aberto no mundo (COSTA; KURAMOTO; LEITE, 2013, p. 134-135; PARCKER; MENEGHINI 2014, p. 19).

Dado, então, o pioneirismo brasileiro na implantação dessas ações, em um período de expansão do parque científico e tecnológico brasileiro e da produção científica do país, conforme expõem Costa, Kuramoto e Leite (2013), o presente trabalho tem como ponto de partida as seguintes questões de pesquisa: passadas quase duas décadas desde a Declaração de Budapeste, os pesquisadores no Brasil teriam aderido ao AA como um meio de divulgar suas pesquisas? Características pessoais e acadêmicas, como gênero, região geográfica da instituição de vínculo e grande área de atuação dos pesquisadores, influenciam na adesão ao AA?

O estudo, que é de caráter quantitativo e descritivo, tem, assim, a proposta de, a partir da produção científica brasileira em periódicos científicos, avaliar o nível de adesão de pesquisadores brasileiros aos periódicos de AA. Busca-se com tal estudo focar um grupo seleto de pesquisadores, os que receberam Bolsas de Produtividade em Pesquisa (PQ) 1A do Conselho Nacional de Desenvolvimento Científico e Tecnológico (CNPq), considerados aqui como 
a elite de pesquisadores da ciência brasileira, uma vez que são reconhecidos como pesquisadores experientes, bem posicionados na carreira acadêmica e de grande prestígio dentro e, na maior parte dos casos, fora da comunidade científica brasileira.

No próximo tópico, apresenta-se o contexto teórico da natureza e dos marcos do AA e o papel central das bolsas de produtividade em pesquisa do CNPq para a estruturação da ciência brasileira.

\section{Contexto teórico do estudo}

O movimento de acesso aberto surge formalmente em 2002 com a Declaração de Budapeste, na qual se estabelece a Iniciativa de Acesso Aberto, Budapest Open Access Initiative (BOAI), seguida por outras declarações e manifestos igualmente importantes para o amadurecimento dos debates e aprimoramento de estratégias para o pleno desenvolvimento do AA no mundo (OPEN ACCESS 2020, 2019).

Duas estratégias foram estabelecidas na Declaração de Budapeste: a via verde, que se alcança pelo depósito de trabalhos completos em repositórios, e a via dourada, que se alcança por meio de uma nova geração de periódicos autossustentáveis e sem cobranças de taxas. Ambas foram reafirmadas no documento conhecido como BOAI 10 com nova denominação: acesso aberto verde e acesso aberto dourado, respectivamente. São estratégias que sugerem que a literatura científica pode estar "ao alcance dos próprios acadêmicos, imediatamente, sem a necessidade de aguardar por mudanças operadas pelos mercados ou pela legislação" (OPEN SOCIETY INSTITUTE, c2001). Porém, a partir de 2012, o movimento do AA sofre com diversos reveses, que vão desde o Relatório Finch, em 2012, que recomendou o financiamento do acesso aberto dourado por meio da cobrança de taxas de processamento de artigos, Article Processing Charge (APC), até o surgimento de periódicos predatórios (HARNAD, 2012a; BARTHOLOMEW, 2014).

Apesar dos grandes avanços trazidos pelo AA para todo o processo de comunicação científica, os desafios para a sua plena adoção são igualmente enormes, distribuídos em dimensões políticas, econômicas, sociais, históricas, 
institucionais, entre outras. Nesta seção, são destacadas algumas dessas questões que resultaram em tensões em torno do acesso aberto dourado e, posteriormente, a elite brasileira de pesquisadores é caracterizada dentro de um quadro teórico pautado em conceitos desenvolvidos por Bourdieu (2004).

\subsection{Acesso aberto dourado e o novo modelo de negócios}

$\mathrm{O}$ advento dos periódicos eletrônicos e a perspectiva de diminuição dos custos de produção colocaram em xeque a cobrança exorbitante das assinaturas ou mesmo a sua necessidade, provocando grande reação das editoras comerciais. Um dos principais argumentos que os editores comerciais aduziam, segundo Odlyzko (c1999), era a necessidade de cobrir os custos de preparação dos originais para publicação, mesmo em ambiente digital. A posição das editoras contrasta com a defesa dos representantes do AA, que verificaram, em vários estudos, tais como o de Solomon (2006) e o de Swan (2016), que os custos de publicação e de disseminação são menores; Solomon (2006) apresenta as estratégias para criar um periódico de acesso aberto sustentável e Swan (2016) apresenta os tipos de custos dos modelos baseados em assinaturas, em APCs e no acesso aberto verde e dourado.

Após quase vinte anos desde o surgimento do movimento do acesso aberto, a batalha dos editores comerciais não só continua, mas se intensifica a partir de algumas medidas e iniciativas tanto do setor editorial privado como também do setor público. Kuramoto (2012), ao discorrer sobre o Relatório Finch, afirma que tais iniciativas são o resultado do acirramento de forças entre agentes econômicos e políticos no Reino Unido que se beneficiaram de um modelo de negócios em que o autor paga para publicar. O Relatório Finch, publicado em 2012, recomendou que fossem cobradas APCs ao autor pela publicação em AA para garantir a sustentabilidade dos periódicos, arcando, assim, não apenas com os custos da disponibilização, mas também da produção, diagramação e revisão, de forma perpétua, de um artigo online. Esses argumentos foram rapidamente difundidos e aplicados pelo Reino Unido, inspirando outros países, especialmente os países sedes de editoras comerciais 
que concentram os títulos de "periódicos de elite" do mundo (LARIVIÈRE; LOZANO; GINGRAS, 2014).

O modelo proposto no Relatório Finch, ou seja, aquele em que o autor paga para publicar, não é novidade e está presente desde o início do movimento de acesso aberto em algumas iniciativas, tais como a Public Library of Science (PloS). De acordo com Michael Eisen (2012), cofundador da Public Library of Science (PloS), a taxa diminuiria, tornando-se quase imperceptível com o tempo. Porém, a taxa persiste até hoje em valores atualizados e competitivos. Importante ressaltar que o estudo de Khoo (2019) demonstrou que não há nenhuma evidência de que autores evitem periódicos que adotam APCs; em outras palavras, essas taxas, não parecem ser um empecilho para a escolha dos periódicos.

É fato que a APC garantiu as condições necessárias para que a indústria de publicações científicas se apropriasse do AA por meio de práticas típicas de oligopólios, cobrando valores exorbitantes que custam £1,000 ou mais (HARNAD, 2012b) para publicar somente um artigo. Esses valores ganham outra dimensão quando se trata de países periféricos, mesmo quando estes se beneficiam de taxas reduzidas. Nassi-Calò (2016), por exemplo, cita um estudo que estima que a Índia invista em média o valor considerável de US\$ 2,4 milhões em APCs por ano.

Os modelos de negócios bem-sucedidos dos oligopólios estão baseados fortemente em duas estratégias: uma em que as editoras lucram com cada artigo publicado em AA nos seus periódicos por meio do pagamento das APCs pelo autor (ou pela instituição de origem, ou pelo órgão de fomento) e outra sustentada pelas assinaturas dos periódicos, caracterizando, assim, o que Harnad (2012a) denominou de "acesso aberto híbrido".

Na Europa, várias iniciativas vêm reforçando a ideia de que as pesquisas financiadas com recursos públicos devem ser difundidas amplamente por meio do AA, fortalecendo os ideais preconizados pelo movimento. Príncipe e outros (2013) elencaram diversas dessas iniciativas europeias, que vão desde as diretrizes de 2007 do Conselho Europeu de Investigação para o Acesso Aberto e o projeto-piloto de AA do $7^{\circ}$ Programa-Quadro criado em 2008 até o novo 
programa-quadro de financiamento europeu para investigação e inovação criado em 2014, nomeado Horizon 2020. Mais recentemente, essa mesma ideia foi reforçada pelo Plano S, uma iniciativa europeia criada em setembro de 2018 por uma coligação internacional de agências de fomento, denominada cOAlition $S$, com o objetivo de acelerar a transição para o AA. Tal iniciativa prevê que, partir de 2021, todas as publicações científicas resultantes de pesquisa financiada com recursos públicos deverão ser publicadas em periódicos ou plataformas de AA, garantindo o acesso pleno e imediato a essas publicações (RODRIGUES, 2019; SCHILTZ, 2018).

A essa iniciativa soma-se outra, a Open Access 2020 (OA 2020), que está sediada na Max Planck Digital Library, na Alemanha, desde 2015, e que reúne mais de 130 representantes de órgãos de pesquisa e fomento também comprometidos em acelerar a transição para o AA (OPEN ACCESS 2020, 2019).

É neste cenário de combate que o movimento de acesso aberto resiste e cresce, mesmo que mais lentamente do que o desejável. Dados do Diretório de Periódicos de Acesso Aberto - Directory of Open Access Journals (DOAJ) do ano de 2019 (DOAJ, 2019)demonstram esse avanço: em 2003, quando o DOAJ foi lançado, o diretório listava 300 títulos em AA; em junho de 2019, o diretório indexava mais de 13.300 títulos, dos quais, é importante ressaltar, quase $25 \%$ cobrava APC. A capilaridade do Movimento é visível quando se examina a origem dos títulos listados no DOAJ: são 130 países, de todas as regiões do mundo. O Brasil tem uma posição de destaque no diretório: em 2019, contava com 1.324 títulos, ocupando a terceira colocação na classificação do DOAJ dos países com o maior número de títulos indexados, atrás somente do Reino Unido, com 1.441 títulos, e da Indonésia, com 1.435 títulos (DOAJ, 2019).

Essa posição de destaque parece ser resultado, conforme visto, de algumas iniciativas nacionais ao longo das últimas décadas tais como aquelas lideradas pela Biblioteca Regional de Medicina (BIREME) e pelo IBICT. Ações mais recentes, especialmente da CAPES e da Fundação de Amparo à Pesquisa do Estado de São Paulo (FAPESP), fortaleceram ainda mais o AA no Brasil. A CAPES se tornou signatária, em 2017, da Declaração da OA 2020 (OPEN 
ACCESS 2020, 2019) e do Plano S, apoiado pelo cOAlition $S$ em defesa da conversão do modelo de negócios de assinaturas para o AA em larga escala sem pagamentos.

A FAPESP, segundo Marques (2019), instituiu, a partir da Portaria CTA n. 01/2019, a Política para Acesso Aberto às Publicações Resultantes de Auxílios e Bolsas, incentivando que as publicações oriundas das pesquisas financiadas por essa fundação estejam imediatamente em AA por meio de depósito do artigo em um repositório público ou de sua publicação em periódico SciELO.

O caminho destas iniciativas, ações e estratégias tem mantido a força e vitalidade do AA no mundo e, em especial, no Brasil.

\subsection{Capital simbólico dos bolsistas de produtividade do CNPq}

No Brasil, algumas agências estaduais e federais financiam pesquisas por meio de diversas modalidades de bolsas e auxílios. Dentre essas agências, o CNPq, criado em 1951, concede uma modalidade de bolsa individual, a bolsa de produtividade em pesquisa, a pesquisadores que se destacam entre seus pares, de forma a "incentivar o aumento da produção científica, tecnológica e inovação de qualidade" (FIOCRUZ, 2019, doc. não paginado). A bolsa PQ divide-se em três categorias, Sênior (PQ-SR), PQ-1 (subdividida nos níveis A, B, C e D) e PQ-2 (CNPQ, 2015), e sua concessão é baseada em exigências distintas, conforme resolução contida no documento (BRASIL, 2015).

Para a categoria PQ 1A, de interesse do presente estudo, além da exigência de oito anos de conclusão do doutorado, os candidatos são avaliados a partir da qualidade e conjunto da obra. Em 2003, foi incluído nas bolsas PQ em nível 1A e 1B o adicional de bancada (grant), conforme Instrução de Serviço ${ }^{\circ}$ 004/2003 sobre a Bolsa de Produtividade em Pesquisa, IS-004/2003 (BRASIL, 2003), para garantir as despesas de custeio dos projetos de pesquisa. No ano seguinte, o benefício se estendeu a todos os pesquisadores da categoria 1, agregando maior valor aos recursos recebidos.

A grande concorrência e, ao mesmo tempo, o número reduzido de bolsas de produtividade fazem dessas bolsas, especialmente as da categoria PQ 1A, um 
símbolo de distinção, ou seja, uma espécie de chancela institucional que reconhece e torna público o mérito e o destaque da pesquisa conduzida pelo/a pesquisador/a contemplado/a. Esse tipo de recompensa caracteriza a ciência contemporânea, estruturando-a e fazendo dela um espaço de disputa no qual cada agente busca atrair e concentrar sobre si certos tipos de distinções que possam lhe assegurar ou conferir autoridade no campo e, consequentemente, determinar a sua posição nele. Sobre esse cenário, Bourdieu (2004) introduz os conceitos de capital científico puro e de capital científico institucional, que são tipos específicos de prestígio comuns na ciência: o primeiro tem origem no reconhecimento pelos pares e, portanto, se relaciona, principalmente, com as publicações e a repercussão delas no meio científico; o segundo tem um caráter mais político e diz respeito aos cargos que os pesquisadores ocuparam e às suas indicações ao longo da sua trajetória no campo.

Dado que a publicação em periódicos é um mecanismo que pode levar a um acúmulo de capital científico puro, é possível inferir que quanto maior o prestígio do periódico, maior será o capital acumulado, o que pode ser traduzido como maior sucesso na carreira. É relevante destacar que dentre os periódicos de maior prestígio estão aqueles editados e gerenciados por oligopólios formados em torno de algumas empresas da indústria editorial, como Elsevier e Wiley, que publicam títulos como The Lancet e A Cancer Journal for Clinicians, respectivamente, com alto fator de impacto e com acesso restrito. Esses aspectos têm se mostrado de grande valor e importância no momento da escolha do periódico no qual os pesquisadores publicam suas pesquisas, tal como sugerem os estudos de Gordon (1984), Starbuck (2005), Nicholas e outros (2017) e Harley e colaboradores (2006).

Em países emergentes como o Brasil, esses aspectos se agravam com o desconhecimento das práticas de acesso aberto. O estudo de Furnival e Guirra (2017), por exemplo, verificou que um grupo de pesquisadores brasileiros da área de engenharia desconhece o caminho complementar de arquivar um artigo em repositório institucional mesmo que o artigo esteja publicado em periódico de alto fator de impacto e com APC, após o término do tempo de embargo da editora, conforme a estratégia expressa na Declaração de Budapeste, de 2002. 
Esse desconhecimento pode ser entendido como um grande obstáculo ao acesso aberto pois promove o pagamento de assinaturas de periódicos.

A despeito da situação observada por Furnival e Guirra (2017), alguns estudos recentes mostram que a fração da produção científica brasileira publicada em periódicos de acesso aberto pode variar muito. Enquanto Archambault e colaboradores (2014) identificaram que 66\% da produção brasileira no período de 2008 a 2013 estavam acessíveis gratuitamente online, Pavan e Barbosa (2018) mostraram que esse percentual foi de 26\% em 2016. Muito embora as variações sejam resultado das diferenças do período considerado na análise e das fontes de dados utilizadas, os dados sugerem a necessidade de investigar esse tema a partir de diferentes dimensões e segmentos da comunidade científica.

Essa é, portanto, a proposta do presente trabalho, que se concentra no segmento de bolsistas de produtividade 1A aqui denominado de elite brasileira de pesquisadores. Trata-se de um segmento da comunidade científica brasileira com alto nível de capital científico reconhecido pela excelência de seu desempenho, pela liderança na área e influência no estabelecimento dos critérios de produção e de avaliação em seus campos de atuação.

\section{Procedimentos metodológicos}

Todos os dados apresentados no presente estudo, de caráter quantitativo e descritivo, foram obtidos diretamente da Plataforma Lattes e do DOAJ, conforme se descreve a seguir.

Primeiramente, foram identificados os bolsistas de produtividade de nível 1A (PQ 1A) do CNPq em sua totalidade, com bolsa ativa de 2016, na Plataforma Lattes, gerando-se uma lista nominal de pesquisadores. Em seguida, foram extraídos os dados sobre a produção científica de cada bolsista. Esse processo foi realizado por meio da ferramenta scriptLattes (MENA-CHALCO; CESAR-JÚNIOR, 2009). Foi delimitado o período de 2000 a 2015, distribuído em quadriênios, como recorte das produções científicas dos pesquisadores bolsistas PQ 1A de 2016 para que fossem analisadas séries históricas a partir dos estágios embrionários do AA. 
A coleta de dados pessoais e acadêmicos e de dados de produção dos pesquisadores bolsistas, neste caso, somente artigos publicados em periódicos científicos, foi realizada em maio de 2016. Todos os dados foram organizados em uma planilha de Excel. Naquele ano, a Plataforma Lattes registrava o total de 116.427 pesquisadores doutores, dos quais 14.489 (12,5\%) eram bolsistas de produtividade em pesquisa nas diversas modalidades do CNPq. Deste total, 1.205 eram pesquisadores bolsistas PQ 1A, representando, assim, 8,3\% do total dos pesquisadores bolsistas.

Paralelamente, em outra planilha foram compilados os dados referentes aos títulos de periódicos de acesso aberto, identificados e caracterizados como tal pelo diretório DOAJ. Dentre os dados coletados, incluíram-se o International Standard Serial Number (ISSNs) e o Eletronic International Standard Serial Number (e-ISSNs), país da publicação, ano de fundação, cobrança ou não de APC, etc.

Em seguida, para cada artigo publicado de autoria dos bolsistas PQ 1A, foi indicado o tipo de acesso ao periódico em que o artigo foi publicado: restrito ou aberto. Esse processo foi realizado de forma automatizada, por meio da comparação entre a planilha com a produção dos pesquisadores, construída a partir da Plataforma Lattes, e a planilha com a lista dos periódicos de acesso aberto, obtida no DOAJ, incluindo os periódicos que cobram APC. O cruzamento dos dados baseou-se no ISSN (ou e-ISSN), presente nas duas planilhas (na da produção dos bolsistas e na dos periódicos). Em alguns casos, seja devido a mudança do ISSN, seja devido a erros de digitação no cadastramento do ISSN ou e-ISSN no Currículo Lattes, foi necessário usar o título completo do periódico para verificar cada produção nas respectivas planilhas. Dessa forma, todas as publicações cujo título de periódico constava nas duas planilhas foram consideradas de AA.

Após o cruzamento de dados descrito acima, uma terceira (e última) planilha, também em formato Excel, foi criada contendo, além dos dados pessoais e acadêmicos, os dados de produção dos pesquisadores bolsistas com a indicação de acesso aberto ou restrito atribuída a cada artigo. Para o presente estudo, foram selecionadas as variáveis gênero, região geográfica e grande área 
para descrever a adesão dos bolsistas de produtividade PQ 1A aos periódicos de acesso aberto.

Para o cálculo da faixa de adesão, levou-se em consideração, para cada pesquisador/a, a fração de artigos que ele/a publicou em periódicos de acesso aberto em relação ao total de artigos que ele/ela publicou em um quadriênio, por exemplo, 2000-2003. Este mesmo cálculo foi repetido para os cada um dos quadriênios. Desta forma, dividiu-se a adesão em cinco categorias:

a) muito baixa: $0 \%$ a $20 \%$;

b) baixa: $20,1 \%$ a $40 \%$;

c) média: $40,1 \%$ a $60 \%$;

d) alta: $60,1 \%$ a $80 \%$;

e) muito alta: $80,1 \%$ a $100 \%$.

\section{Resultados}

Os resultados sobre da análise de adesão da elite brasileira de pesquisadores estão apresentados em dois blocos: o primeiro apresenta-uma análise mais geral sobre as faixas de adesão ao AA por quadriênio, enquanto o segundo bloco apresenta o efeito das variáveis na faixa de adesão ao AA.

\subsection{Visão geral das faixas de adesão ao acesso aberto}

Para a análise da adesão ao AA, foi considerada a fração de artigos publicados em periódicos de acesso aberto, conforme listagem do DOAJ, de um pesquisador bolsista em relação ao total de artigos publicados por ele. Exemplo: o pesquisador A publicou 1 um artigo em AA e 19 artigos em acesso restrito. Ou seja, ele possui 20 artigos publicados. Sendo $1 / 20$ igual a 5\%, a faixa correspondente deste pesquisador é de 0 a $20 \%$ de adesão, isto é, muito baixa adesão.

O Gráfico 1 apresenta a distribuição dos pesquisadores bolsistas PQ 1A segundo a faixa de adesão ao AA e o quadriênio em que a produção foi publicada. 
Gráfico 1 - Pesquisadores bolsistas PQ 1A do CNPq (\%), segundo a faixa de adesão aos periódicos de acesso aberto, por quadriênio.

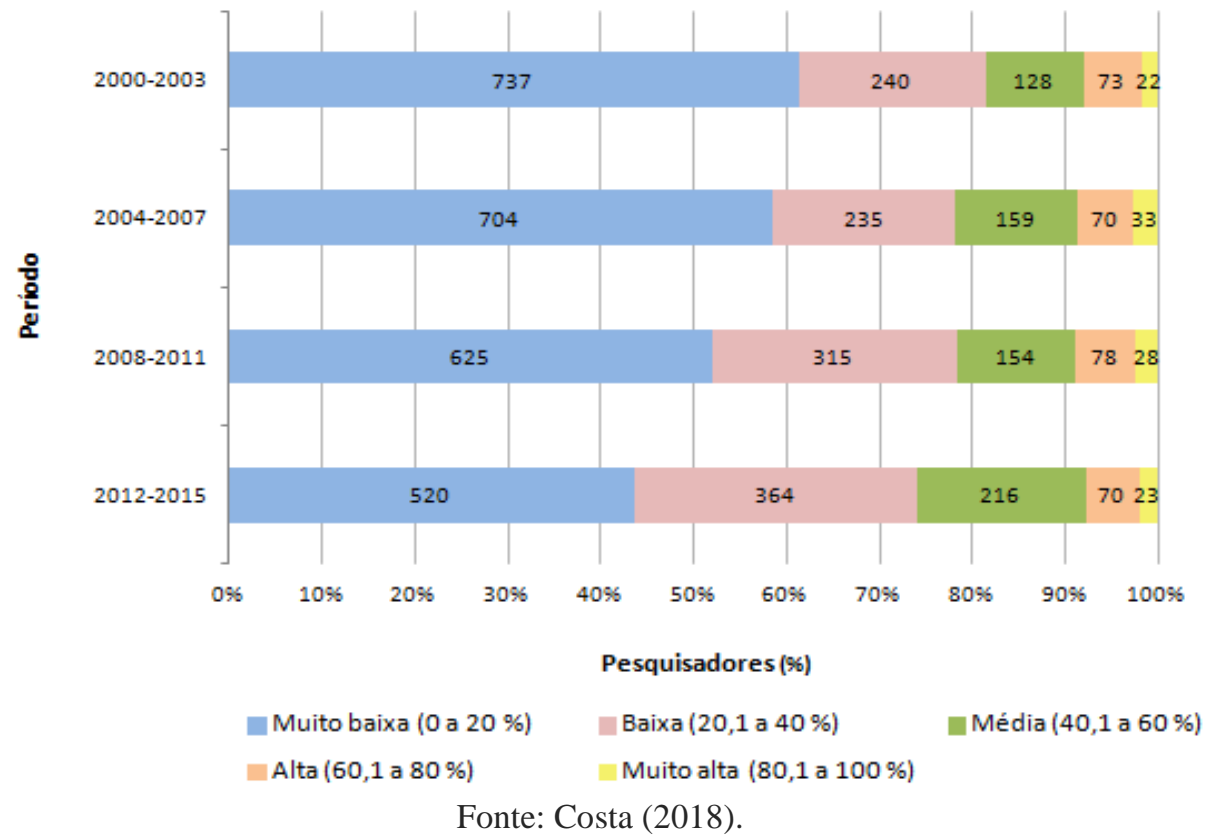

O total de pesquisadores em cada faixa de adesão por quadriênio está representado pelos números arábicos. Observa-se que a grande maioria dos pesquisadores apresenta um perfil com muito baixa ou baixa adesão aos periódicos de acesso aberto, faixas que concentram de $75 \%$ a $80 \%$ dos bolsistas PQ 1A ao longo dos quadriênios.

Importante ressaltar que, a despeito desse dado alarmante, é clara a redução no número e no percentual de pesquisadores bolsistas da faixa de muito baixa adesão, que passou de 737 pesquisadores (62\%) no quadriênio 2000-2003 para 520 pesquisadores (44\%) no quadriênio 2012-2015. A redução observada nesta faixa acompanha o aumento em outras duas faixas, de baixa e média adesão, o que sugere que, nos últimos anos, parte desse grupo seleto de pesquisadores mudou sua práxis e incorporou mais os periódicos de acesso aberto como meio de difundir suas pesquisas.

As mudanças descritas nas faixas de menor adesão não se refletiram, no entanto, em um aumento quantitativo (ou de percentual) de pesquisadores na faixa de alta ou muito alta adesão, que somam cerca de 100 pesquisadores por quadriênio, ou $10 \%$ do total, classificados com esses perfis. 
$\mathrm{Na}$ próxima seção, está detalhada a adesão da elite brasileira de pesquisadores ao AA, na sua relação com gênero, região geográfica e grande área de conhecimento dos pesquisadores PQ 1A do CNPq.

\subsection{Efeito das variáveis nas faixas de adesão ao acesso aberto}

Para melhor entender a adesão dos pesquisadores bolsistas PQ 1A, optamos por deter nos extremos do indicador de adesão, ou seja, nos pesquisadores com muito baixa e com alta/muito alta adesão, buscando evidenciar possíveis relações desses perfis com outras variáveis. A primeira variável a ser analisada diz respeito ao gênero dos pesquisadores nestas nessas duas faixas de adesão, o que se apresenta no Gráfico 2.

Gráfico 2 - Pesquisadores bolsistas PQ 1A do CNPq (\%) com muito baixa adesão (A) e com alta/muito alta adesão (B) aos periódicos de acesso aberto, por gênero, nos quadriênios

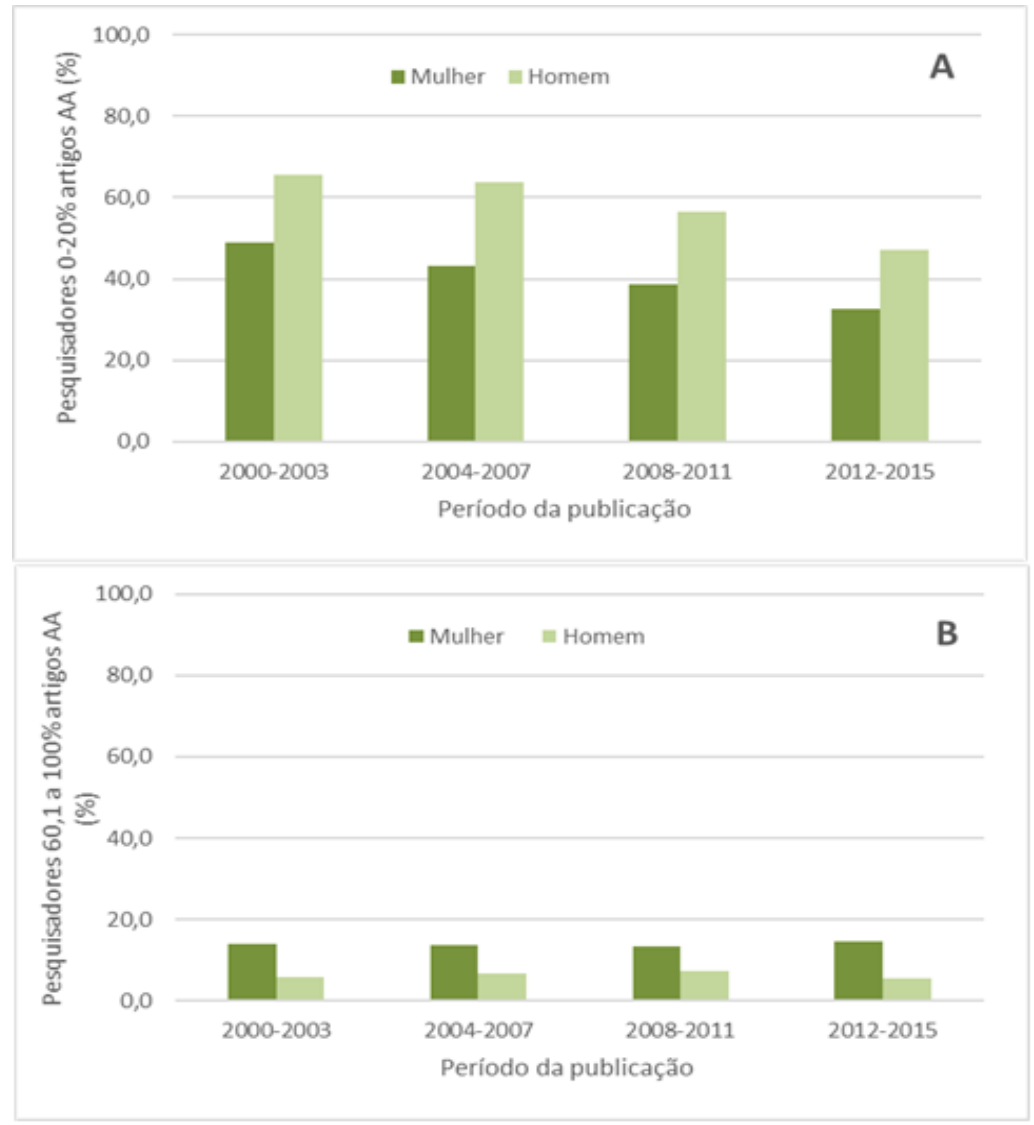

Fonte: Costa (2018).

É possível observar que entre os pesquisadores com perfil de muito baixa adesão (0-20\%), os homens predominam. Porém, entre aqueles classificados 
como de alta/muito alta adesão (60,1 a 100\%), observamos que as mulheres predominam. Isto significa que mulheres publicam mais em AA que os homens, ou seja, 14 em cada 100 mulheres publicam nesta faixa de adesão, enquanto cerca de 6 homens em cada 100 têm o mesmo desempenho.

Importante destacar que, dentre os 1.205 bolsistas PQ 1A, as mulheres somam 301, ou seja, $25 \%$ da população estudada, uma fração que está abaixo daquela observada para mulheres bolsistas em qualquer modalidade de bolsa PQ/CNPq (LIMA; BRAGA; TAVARES, 2015), que foi $36 \%$ para o ano de 2014, e daquela observada para mulheres pesquisadoras cadastradas no Diretório de Grupos de Pesquisa, que chega a 50,4\% (BRASIL, 2019a). Observa-se, portanto, que as mulheres estão sub-representadas na população de estudo. A despeito disso, proporcionalmente, elas mostram maior adesão ao AA que seus colegas bolsistas homens.

Uma segunda variável, a região geográfica da instituição de vínculo dos pesquisadores, foi analisada ao longo dos quadriênios, tal como se apresenta no Gráfico 3. 
Gráfico 3 - Pesquisadores bolsistas PQ 1A do CNPq (\%) com muito baixa adesão (A) e com alta e muito alta adesão (B) ao acesso aberto, por região geográfica da instituição de vínculo, nos quadriênios

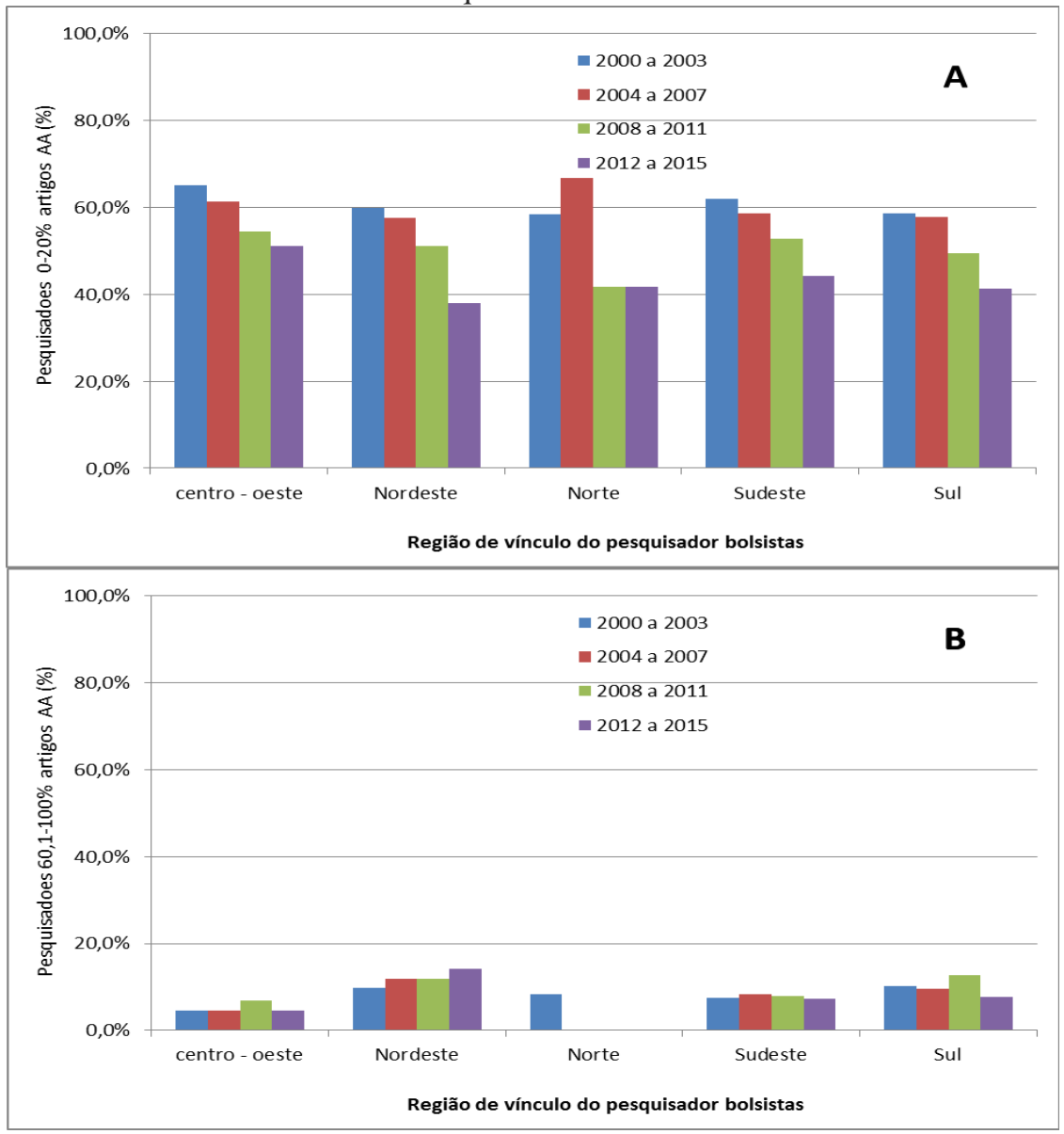

Fonte: Costa (2018).

O Gráfico 3A demonstra que a fração de pesquisadores com perfil de muito baixa adesão (de 0 a 20\%) praticamente não difere entre as regiões. Observa-se também que a fração de pesquisadores com esse perfil diminuiu ao longo dos quadriênios.

No gráfico 3B, observa-se uma ligeira tendência a favor de pesquisadores nas faixas de maior adesão (alta ou muito alta) oriundos do Nordeste, publicando mais em periódicos de acesso aberto que os pesquisadores das demais regiões. Esse dado é relevante se considerarmos que pesquisadores bolsistas PQ 1A da região Nordeste representam cerca de $8 \%$ dos pesquisadores analisados (92 pesquisadores).

A grande área do conhecimento foi a terceira e última característica analisada com pesquisadores na faixa de muito baixa (0-20\%) e de alta ou muito 
alta adesão $(60,1 \%$ a 100\%) aos periódicos de acesso aberto, conforme demonstra o gráfico 4 .

Gráfico 4 - Pesquisadores bolsistas PQ 1A do CNPq (\%) com muito baixa adesão (A) e com alta e muito alta adesão (B) aos periódicos de acesso aberto, por grande área do conhecimento, nos quadriênios

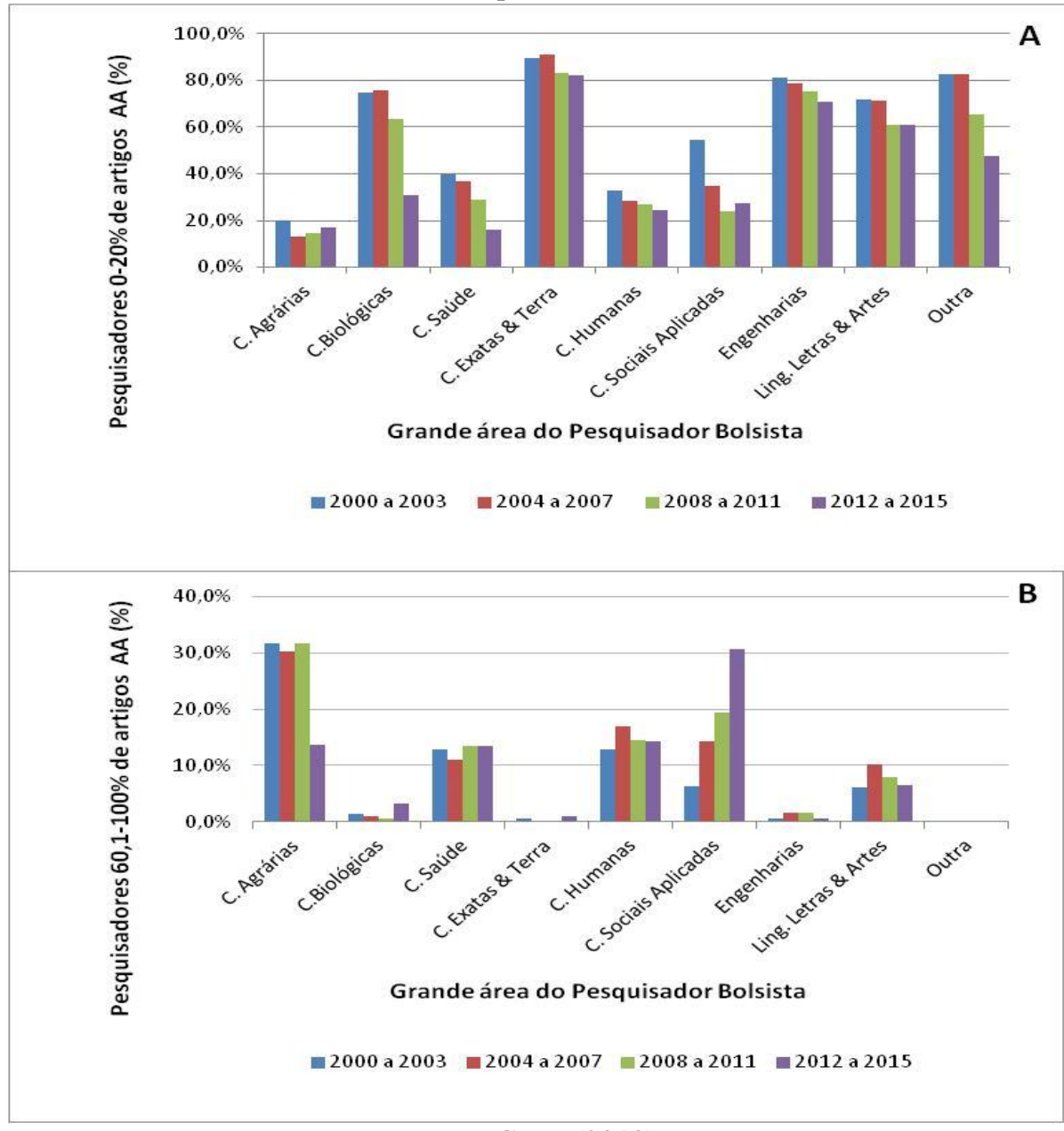

Fonte: Costa (2018).

No gráfico 4A, observa-se que, entre os pesquisadores com perfil de muito baixa adesão, predominam aqueles das Ciências Exatas e da Terra, aqueles de outra grande área não especificada, aqueles das Engenharias, aqueles das Ciências Biológicas e aqueles da grande área de Linguística/Letras/Artes, decrescentemente. Já na faixa da alta ou muito alta adesão (Gráfico 4B), pesquisadores das Ciências Agrárias e das Ciências Sociais Aplicadas predominam. Nesta última grande área, observa-se um evidente crescimento na fração de pesquisadores que publicam nesta faixa de adesão no decorrer dos quadriênios. 
Vale ressaltar que entre as grandes áreas de maior adesão, estão não apenas aquelas que tradicionalmente têm maior presença de mulheres (Ciências Sociais e Ciências Humanas), mas também outras em que há equilíbrio de gênero (Ciências da Saúde) e em que elas são minoria (Ciências Agrárias), tal como descrito pelo Ministério da Ciência, Tecnologia, Inovações e Comunicações do Brasil, (BRASIL, 2019b). Além disso, a grande área da Linguística, que conta com a maior fração de mulheres bolsistas PQ 1A $(58,8 \%)$, tem, proporcionalmente, menor adesão que as mencionadas anteriormente. Conjuntamente, estes dados sugerem que a distribuição dos pesquisadores com maior e com menor adesão ao AA não é dependente da variável gênero dos pesquisadores.

\section{Considerações finais}

Ao avaliar-se o nível de adesão da totalidade dos bolsistas de produtividade PQ $1 \mathrm{~A}$ do $\mathrm{CNPq}$ aos periódicos de AA, foi observado que menos de $10 \%$ dos pesquisadores foram classificados no nível de adesão alto ou muito alto, uma fração que não se alterou ao longo do período. Este dado chama atenção porque demonstra que a maior parte da elite brasileira de pesquisadores está publicando preferencialmente em periódicos de acesso restrito, conforme pode ser constatado a partir do Gráfico 1.

Por outro lado, a análise mais geral revelou que, ao longo dos quadriênios, houve uma redução nas faixas de pesquisadores de muito baixa e de baixa adesão aos periódicos de AA, ou seja, houve uma redução nas faixas de pesquisadores cuja produção publicada em periódicos de acesso aberto representa até $40 \%$ de sua produção publicada total. Apesar de essa redução sinalizar um número crescente de adesão de pesquisadores aos periódicos de AA, a concentração de pesquisadores nessas faixas ainda é muito alta, representando cerca de $80 \%$ da população estudada (Gráfico 1).

A segunda parte das análises procurou determinar se as características relativas ao gênero, à região geográfica e à grande área do conhecimento associadas à população estudada poderiam ou não explicar os graus de adesão ao AA tanto na faixa de muito baixa adesão como nas faixas de alta e muito alta 
adesão. Destas características, gênero e grande área indicam variações mais expressivas no padrão de adesão ao AA.

Quanto ao gênero dos pesquisadores, os resultados indicam que, proporcionalmente, os homens em relação às mulheres estão mais presentes na faixa de muito baixa adesão, enquanto as mulheres em relação aos homens estão mais nas faixas de alta e muito alta adesão (Gráfico 2). Sobre esse resultado, chama a atenção o fato de que o número de pesquisadores bolsistas PQ 1A do gênero masculino é três vezes maior do que o de pesquisadoras bolsistas PQ 1A do gênero feminino: 904 homens e 301 mulheres (COSTA, 2018).

Já a análise sobre as grandes áreas revelou que pesquisadores de Ciências Exatas e da Terra, de outras grandes áreas, das Engenharias, das Ciências Biológicas e das áreas de Linguística/Letras/Artes predominam na faixa de muito baixa adesão ao AA, enquanto pesquisadores das Ciências Agrárias e das Ciências Sociais Aplicadas prevalecem nas faixas da alta ou muito alta adesão (Gráfico 4). Esses resultados, de certa forma, contrastam com os resultados do estudo conduzido por Archambault e outros (2014), que observaram que as áreas de Artes Visuais (23\%), Comunicação (27\%), Historia (30\%), Psicologia \& Teologia (30\%) e Engenharia (30,2\%) são as que apresentam a menor fração de publicação em AA. Além disso, os autores mostraram que a Biologia (58\%) e a Biomédica (62\%) estão entre as áreas com maior fração de publicação em AA.

Muito embora o trabalho de Archambault e colaboradores (2014) analise publicações do período de 1996 a 2013, indexadas na base Scopus, e considere como de AA qualquer publicação acessível gratuitamente online, o que difere do conceito de AA usado no presente trabalho (concentrado nos periódicos listados no DOAJ), a ampla cobertura de tempo, de países, como o Brasil, e de área do conhecimento, com análise de milhões de publicações, releva dados úteis e que suscitam reflexões sobre o perfil encontrado para a elite brasileira de pesquisadores.

Em resumo, o conjunto de dados acerca da produção dos pesquisadores de maior prestígio da ciência brasileira, os pesquisadores bolsistas PQ 1A do CNPq, e acerca da adesão desses pesquisadores aos periódicos de acesso aberto 
permite inferir que, a despeito do movimento crescente dos pesquisadores em direção ao AA, verificado neste estudo, esse grupo seleto de pesquisadores ainda prioriza a publicação em periódicos científicos de acesso restrito. Esse mecanismo parece garantir a eles maior reconhecimento e legitimação de seus pares, que se expressam em maior autoridade e poder dentro do campo em que atuam. A opção desses pesquisadores pela publicação em periódicos de acesso restrito, especialmente naqueles sob o controle das grandes corporações editoriais que são, na maior parte dos casos, de grande influência e visibilidade, pode ser entendida como uma estratégia para assegurar o acúmulo de capital científico e, consequentemente, para manter posição na estrutura de prestígio no campo.

Dados os modelos de avaliação das agências brasileiras de fomento à pesquisa, que se pautam, em muitos casos, por indicadores quantitativos de desempenho, visibilidade e impacto, como fator de impacto dos periódicos, é possível inferir que a escolha por periódicos de acesso restrito (os quais, em geral, têm os melhores índices) reforça não apenas a noção de acúmulo de capital científico (BOURDIEU, 2004), mas também a manutenção do status quo deste grupo. É viável, portanto, acreditar que tal escolha seja ao mesmo tempo consequência da pressão das agências e também causa da permanência desse grupo no lugar de maior destaque da ciência brasileira.

Também pode ocorrer que os pesquisadores bolsistas PQ 1A do CNPq prefiram publicar em periódicos de acesso restrito porque, conforme se viu, eles não saibam ou não se interessem por saber o que é um periódico de acesso aberto, e porque desconheçam seus benefícios ou não se interessem por conhecê-los, tal como foi discutido no estudo de Furnival e Guirra (2017) .

Também é possível que nessa escolha os pesquisadores não considerem o acesso aberto como critério, mas o impacto, o prestígio e reconhecimento simbólico do periódico, tal como observaram Boukacem-Zegmouri e colaboradores (2018), que investigaram pesquisadores franceses das áreas exatas.

Certamente algumas questões emergiram a partir dos resultados apresentados neste trabalho, como, por exemplo: por que mais mulheres, 
pesquisadoras bolsistas PQ 1A, que estão em menor proporção que seus colegas homens, buscam os periódicos de AA para publicar? Estariam elas mais sensibilizadas e motivadas? De qualquer forma, o pleno entendimento das motivações desse grupo seleto de pesquisadores ou das razões de as faixas de adesão continuarem baixas não faz parte do escopo deste trabalho, podendo ser objetivo de trabalhos futuros a serem desenvolvidos pelo grupo de autoras do presente estudo.

Para finalizar, é fato que as universidades brasileiras, principalmente as públicas, desempenham um papel fundamental no desenvolvimento científico, tecnológico, social, cultural e econômico do país. Seria salutar que a elite de pesquisadores do país, que estão vinculados principalmente a essas instituições, publicasse em AA em função da capacidade do AA de atrair maior atenção para a pesquisa e para o pesquisador e de aumentar seu impacto. No entanto, a julgar pelo distanciamento que esse grupo de destaque demonstra em relação aos periódicos de acesso aberto, é possível acreditar que somente a implantação de uma política de Estado, em um esforço coletivo, coordenado pelos principais atores envolvidos, incluindo as diferentes agências de fomento, levará a uma mudança de rumo em direção a uma efetiva adesão ao AA, o que poderá quiçá resultar em benefícios diretos para muitos setores da sociedade.

\section{Financiamento}

Este trabalho conta com auxílio financeiro do Projeto contemplado com recurso MCTI/CNPq n. 28/2018, Processo n. 434146/2018-8.

\section{Agradecimentos}

Agradecemos ao Prof. Dr. Jesus P. Mena-Chalco que extraiu os dados da Plataforma Lattes dos pesquisadores bolsistas PQ 1A apresentados neste artigo e a Júlio Francisco Costa que auxiliou na sistematização dos dados das tabelas.

\section{Referências}

ARCHAMBAULT, E. et al. Proportion of open access papers published in peer-reviewed journals at the European and world levels-1996-2013.

Montreal: Science-Metrix, 2014. 
ARCHAMBAULT, E. et al. Research impact of paywalled versus open access papers. Copyright, Fair Use, Scholarly Communication, etc., Nebraska, n. 29, p. 1-6, 2016.

BARTHOLOMEW, R. E. Science for sale: the rise of predatory journals. Journal of the Royal Society of Medicine, London, v. 107, n. 10, p. 384-385, Oct. 2014. Disponível em:

https://journals.sagepub.com/doi/10.1177/0141076814548526. Acesso em: 30 set. 2020 .

BJÖRK, B.; HOLMSTRÖM, J. Benchmarking scientific journals from the submitting author's viewpoint. Learned Publishing, Hatfield, v. 19, n. 2, p. 147-155, 2006.

BOUKACEM-ZEGMOURI, C. et al. French publishing attitudes in the open access era: the case of mathematics, biology, and computer science. Learned Publishing, Hatfield, v. 31, p. 345-354, 2018.

BOURDIEU, P. Os usos sociais da ciência: por uma sociologia clínica do campo científico. São Paulo: Editora UNESP, 2004.

BRASIL. Ministério da Ciência, Tecnologia, Inovações e Comunicações. Conselho Nacional de Desenvolvimento Científico e Tecnológico.Chamada CNPq No 09/2018 Bolsas de Produtividade em Pesquisa - PQ. Brasília, DF: CNPq, 2018. Disponível em: http://cnpq.br/chamadaspublicas?p_p_id=resultadosportlet_WAR_resultadoscnpqportlet_INSTANCE_0 ZaM\&filtro=abertas\&detalha $=$ chamadaDivulgada\&idDivulgacao=8022. Acesso em: 1 nov. 2019.

BRASIL. Ministério da Ciência, Tecnologia, Inovações e Comunicações. Conselho Nacional de Desenvolvimento Científico e Tecnológico. RN$\mathbf{0 2 8 / 2 0 1 5}$. Bolsas individuais no país. Resolve estabelecer as normas gerais e específicas para as modalidades de bolsas individuais no País relacionadas no Anexo I. Brasília, DF: CNPq, 2015. Disponível em:http://cnpq.br/web/guest/view//journal_content/56_INSTANCE_0oED/10157/2958271?COMPANY_ID=1013 2. Acesso em: 4 nov. 2019.

BRASIL. Ministério da Ciência, Tecnologia, Inovações e Comunicações. Conselho Nacional de Desenvolvimento Científico e Tecnológico. IS004/2003. Bolsa de Produtividade em Pesquisa. Resolve estabelecer os procedimentos para o processo de concessão e implementação da bolsa de Produtividade em Pesquisa, visando ao incentivo à execução de projetos de pesquisa científica e/ou tecnológica. Brasília, DF: CNPq, 2003. Disponível em: http://cnpq.br/view/-/journal_content/56_INSTANCE_0oED/10157/71276. Acesso em: 9 dez. 2019. 
BRASIL. Ministério da Ciência, Tecnologia, Inovações e Comunicações. Brasil: Pesquisadores cadastrados no Diretório dos Grupos de Pesquisa do CNPq por sexo, 2000/2016. In: BRASIL. Ministério da Ciência, Tecnologia, Inovações e Comunicações. Recursos Humanos: indicadores de grupos de pesquisa. Brasília: MCTIC, 2019a. cap. 3.6.2. Disponível em: https://www.mctic.gov.br/mctic/opencms/indicadores/detalhe/Recursos_Human os/RH_3.6.2.html. Acesso em: 8 abr. 2020.

BRASIL. Ministério da Ciência, Tecnologia, Inovações e Comunicações. Brasil: pesquisadores cadastrados no Diretório dos Grupos de Pesquisa do CNPq por grande área e sexo, 2000/2010. In: BRASIL. Ministério da Ciência, Tecnologia, Inovações e Comunicações. Recursos Humanos: indicadores de grupos de pesquisa. Brasília: MCTIC, 2019b. cap. 3.6.4. Disponível em: https://www.mctic.gov.br/mctic/opencms/indicadores/detalhe/Recursos_Human os/RH_3.6.4.html. Acesso em: 8 abr. 2020.

BRASIL. Ministério da Educação. Portaria nº 013, de 15 de fevereiro de 2006. Institui a divulgação digital das teses e dissertações produzidas pelos programas de doutorado e mestrado reconhecidos. Brasília, DF: Ministério da Educação, 2006. Disponível em: https://www.capes.gov.br/images/stories/download/legislacao/Portaria_013_200 6.pdf. Acesso em: 25 jul. 2020.

BRÖCHNER, J.; BJÖRK, B. C. Where to submit? Journal choice by construction management authors, London, v. 26, n. 7, p. 739-749, 2008.

CHEUNG, C. K. Audience matters: a study of how authors select educational journals. Asia-Pacific Education Researcher, Singapore, v. 17, n. 2, p. 191201, 2008.

COSTA, E. H. S. Periódicos de acesso aberto: um canal de disseminação dos pesquisadores bolsistas PQ1A do CNPq? 2018. Tese (Doutorado em Ciência da Informação) - Escola de Comunicação, Universidade Federal do Rio de Janeiro, Rio de Janeiro, 2018.

COSTA, S. M. S.; KURAMOTO, H.; LEITE, F. C. L. Acesso Aberto no Brasil: aspetos históricos. In: RODRIGUES, E.; SWAN, A.; BAPTISTA, A. A. (ed.). Uma década de acesso aberto na UMinho e no mundo. Braga: Publito, 2013. p. 133-150.

DOAJ Directory of Open Access Journals. [S. l.]: DOAJ, c2019. Disponível em: https://doaj.org/. Acesso em: 9 dez. 2019.

EISEN, Michael. The OA Interviews: Michael Eisen, co-founder of the Public Library of Science [Entrevista concedida à] Richard Poynder. Feb. 2012. 19 p. Disponível em: https://www.richardpoynder.co.uk/Eisen_Interview.pdf. Acesso em: 21 jul. 2020. 
FIOCRUZ. Centro de Integração de Dados e Conhecimentos para Saúde. Bolsa de produtividade em Pesquisa (Chamada CNPq 06/2019). Salvador, 2019.

Disponível em: https://cidacs.bahia.fiocruz.br/2019/05/23/bolsas-deprodutividade-em-pesquisa-chamada-cnpq-06-2019/. Acesso em: 21 jul. 2020.

FURNIVAL, A. C.; GUIRRA, D. A. R. As percepções e práticas de publicação em acesso aberto dos pesquisadores de dois programas de pós-graduação em engenharia. Revista Digital de Biblioteconomia e Ciência da Informação, São Paulo, v. 15, n. 2, p. 469-488, 2017.

FURNIVAL, A. C. M.; SILVA-JEREZ, N. S. Percepções de pesquisadores brasileiros sobre o acesso aberto à literatura científica. Informação \& Sociedade: Estudos, João Pessoa, v. 27, n. 2, p. 153-166, 2017.

GORDON, M. D. How authors select journals: a test of the reward maximization model of submission behaviour. Social Studies of Science, Beverly Hills, v. 14, n. 1, p. 27-43, 1984.

GUMPENBERGER, C.; OVALLE-PERANDONES, M. A.; GORRAIZ, J. On the impact of Gold Open Access journals. Scientometrics, Amsterdam, v. 96, n. 1, p. 221-238, 2013.

HARLEY, D. et al. The Influence of academic values on scholarly publication and communication practices. Berkeley: Center for Studies in Higher Education, 2006.

HARNAD, S. OA advocate Stevan Harnad withdraws support for RCUK policy. In: POYNDER, R. Blog Open \& Shut? [S. l.], 26 July 2012a. Disponível em: https://poynder.blogspot.com/2012/07/oa-advocate-stevan-harnadwithdraws_26.html. Acesso em: 10 dez. 2019.

HARNAD, S. Why the UK Should Not Heed the Finch Report. In: TASTE, M. Blog LSE Impact.[S. l.], 4 July 2012b. Disponível em: https://blogs.lse.ac.uk/impactofsocialsciences/2012/07/04/why-the-uk-shouldnot-heed-the-finch-report/. Acesso em: 21 jul. 2020.

KHOMAMI, N. All scientific papers to be free by 2020 under EU proposals: results of research supported by public and public-private funds set to be made freely available to all. The Guardian, [London], 28 May 2016.

KHOO, SY-S. Article processing charge hyperinflation and price insensitivity: an open access sequel to the serials crisis. LIBER Quarterly, Hague, v. 29, n. 1, p. 1-8, 2019.

KURAMOTO, H. O relatório FINCH e suas repercussões. In: KURAMOTO, H. Blog do Kuramoto. [S. l.], 21 ago. 2012. Disponível em: https://kuramoto.wordpress.com/2012/08/21/o-relatorio-finch-e-suasrepercussoes/. Acesso em: 10 dez. 2019. 
LARIVIÈRE, V. G.; LOZANO, A.; GINGRAS, I. Are elite journals declining? Journal of the Association for Information Science and Technology, Hoboken, v. 65, n. 4, p. 649-655, 2014.

LARIVIÈRE, V.; HAUSTEIN, S.; MONGEON, P. The Oligopoly of Academic Publishers in the Digital Era. PLoS One, San Francisco, v. 10, n. 6, p. 1-15, 2015.

LIMA, B. S.; BRAGA, M. L. S.; TAVARES, I. Participação das mulheres nas ciências e tecnologias: entre espaços ocupados e lacunas. Gênero, Niterói, v. 16, n. 1, p. 11-31, 2015.

MARQUES, F. FAPESP lança política para acesso aberto. Pesquisa FAPESP, São Paulo, v. 277, 2019.

MENA-CHALCO, J. P.; CESAR-JÚNIOR, R. M. ScriptLattes: uma ferramenta para extração e visualização de conhecimento a partir de Currículos Lattes.

Journal of the Brazilian Computer Society, [s. l.], v. 15, n. 4, p. 31-39, 2009. Disponível em: http://scriptlattes.sourceforge.net/. Acesso em: 29 out. 2019.

MOORE, G. Survey of University of Toronto Faculty awareness, attitudes, and practices regarding scholarly communication: a preliminary report. Toronto: University of Toronto, 2011.

NASSI-CALÒ, L. Taxas de publicação em Acesso Aberto: nova crise das publicações seriadas? In: SCIELO. Blog SciELO em Perspectiva. São Paulo, 2016. Disponível em: https://blog.scielo.org/blog/2016/11/29/taxas-depublicacao-em-acesso-aberto-nova-crise-das-publicacoesseriadas/\#.Xqiu_ilKjcd. Acesso em: 9 dez. 2019.

NICHOLAS, D. et al. Early career researchers and their publishing and authorship practices. Learned Publishing, Hatfield, v. 30, n. 3, p. 205-217, 2017.

ODLYZKO, A. The economics of electronic journals. In: EKMAN, R.; QUANDT, R. E. (ed.). Technology and scholarly communication. Berkerley: University of California Press, c1999. p. 380-393.

OPEN ACCESS 2020. Expression of interest in the large-scale implementation of open access to scholarly journals. Munich: Max Planck Digital Library, 2019. Disponível em: https://oa2020.org/mission/\#eois. Acesso em: 10 dez. 2019.

OPEN SOCIETY INSTITUTE. Iniciativa de Budapeste pelo Acesso Aberto. Budapest: Open Society Institute, c2001.

PACKER, A. L.; MENEGHINI, R. O SciELO aos 15 anos: raison d'être, avanços e desafios para o futuro. In: PACKER, A. L., et al. (ed.). SciELO - 15 
Anos de Acesso Aberto: um estudo analítico sobre Acesso Aberto e comunicação científica. Paris: UNESCO, 2014. p. 15-28. Disponível em: http://old.scielo.org/local/File/livro.pdf. Acesso em: 4 nov. 2019.

PAVAN, C.; BARBOSA, M. C. Article processing charge (APC) for publishing open access articles: the Brazilian scenario. Scientometrics, Amsterdam, v. 117, n. 2, p. 805-823, 2018.

PEPERMANS, G.; ROUSSEAU, S. The decision to submit to a journal: another example of a valence-consistent Shift? Journal of the Association for Information Science and Technology, Hoboken, v. 67, n. 6, p. 1372-1383, 2016.

PRÍNCIPE, P. et al. Os investigadores em Portugal e a sua relação com o Acesso Aberto à produção científica. In: RODRIGUES, E.; SWAN, A.; BAPTISTA, A. A. (ed.). Uma década de acesso aberto na UMinho e no mundo. Braga: Publito, 2013. p. 173-186.

RODRIGUES, E. Implementação do Plano S: reação do RCAAP e apelo à comunidade. In: UMIC. Blog RCAAP: Repositórios Científicos de Acesso Aberto de Portugal. [S. l.], 2019. Disponível em:

https://blog.rcaap.pt/2019/02/05/implementacao-do-plano-s-reacao-do-rcaap-eapelo-a-comunidade/\#more-3278. Acesso em: 5 nov. 2019.

RODRIGUEZ, J. E. Awareness and attitudes about open access publishing: a glance at generational differences. The Journal of Academic Librarianship, Amsterdam, v. 40, n. 6, p. 604-610, 2014.

SCHILTZ, M. Why Plan S. Bruxelas: Science Europe, 2018. Disponível em: https://www.coalition-s.org/why-plan-s/. Acesso em: 9 dez. 2019.

SCHROTER, S.; TITE, L.; SMITH, R. Perceptions of open access publishing: interviews with journal authors. British Medical Journal, London, v. 330, n. 7494, p. 756, 2005.

SHEIKH, A. Faculty awareness, use and attitudes towards scholarly open access: a Pakistani perspective. Journal of Librarianship and Information Science, London, v. 51, n. 3, p. 612-628, 2017.

SOLOMON, D. J. Strategies for developing sustainable open access scholarly journals. First Monday, Bridgman, v. 11, n. 6, 2006.

STARBUCK, W. H. How much better are the most-prestigious journals? The statistics of academic publication. Organization Science, New York, v. 16, n. 2, p. 180-200, 2005.

SWAN, A. The costs and benefits to the research community of Open Access: a briefing paper. [S. l.]: PASTEUR4OA, Mar. 2016. Disponível em: 
http://www.pasteur4oa.eu/sites/pasteur4oa/files/resource/Costs\%20of\%20OA\% 20final_0.pdf. Acesso em: 8 abr. 2020.

TENOPIR, C. et al. Imagining a gold open access future: attitudes, behaviors, and funding scenarios among authors of academic scholarship. College $\&$

Research Libraries, Chicago, v. 78, n. 6, p. 824-843, 2017.

\title{
Adherence of the Brazilian elite of researchers to open access journals and its relation to gender, region and area of knowledge
}

\begin{abstract}
This study aims to assess the level of adherence the Brazilian elite of researchers to open access journals as a strategy to publish their research. In this study, the 1,205 researchers who received the CNPq PQ 1A productivity scholarship in 2016 are considered as the Brazilian elite. Personal and academic data as well as data of scientific production from these researchers were collected (2000-2015) in the Lattes Platform. Adherence was defined as the fraction of open access articles of each of the 1,205 PQ 1A researchers in relation to the total number of articles they published. Three analyses were conducted to identify the relationship between adherence to open access journals and: (1) the researchers' gender, (2) the geographic region of the researchers' affiliation and (3) their main area of research. Over the four quadrennia, the number of researchers of very low adherence to open access was to decrease, but only $10 \%$ of researchers showed high or very high adherence. Female researchers, researchers from the Northeast region and researchers from the Agricultural and Applied Social Sciences showed greater adherence to open access journals. Adherence to open access journals by the Brazilian elite of researchers, though growing, was still found to be low, a worrying situation, considering that this group is a reference for the Brazilian scientific community.
\end{abstract}

Keywords: Open Access. Scientific journals. Brazilian science. ScriptLattes. Scientific production.

Recebido: 02/01/2020

Aceito: $13 / 04 / 2020$

\section{Como citar}

COSTA, Elaine Hipólito dos Santos; WEITZEL, Simone da Rocha; LETA, Jacqueline. Adesão da elite brasileira de pesquisadores aos periódicos de acesso aberto: a relação com gênero, região geográfica e grande área do conhecimento. Em Questão, Porto Alegre, v. 26, n. 3, p. 15-42, set./dez. 2020. DOI:

https://doi.org/10.19132/1808-5245263.15-42 